\title{
Targeting intermittent hypoxia downstream pathways for biomarker discovery and new treatment perspectives in cutaneous melanoma
}

\author{
Mélanie Minovès, Jean-Louis Pépin and Diane Godin-Ribuot (i) \\ Affiliation: Université Grenoble Alpes, Inserm, CHU Grenoble Alpes, HP2, Grenoble, France.
}

Correspondence: Diane Godin-Ribuot, Université Grenoble Alpes, Inserm, CHU Grenoble Alpes, HP2, 38000 Grenoble, France. E-mail: Diane.RibuotQuniv-grenoble-alpes.fr

@ERSpublications

Targeting the pathways induced by obstructive sleep apnoea-associated intermittent hypoxia could significantly improve biomarker discovery and new treatment perspectives in patients with cutaneous melanoma http://ow.ly/wF3J30nlzL4

Cite this article as: Minovès M, Pépin J-L, Godin-Ribuot D. Targeting intermittent hypoxia downstream pathways for biomarker discovery and new treatment perspectives in cutaneous melanoma. Eur Respir J 2019; 53: 1802444 [https://doi.org/10.1183/13993003.02444-2018].

\section{Obstructive sleep apnoea and melanoma}

In addition to being an independent risk factor for cardiometabolic disease [1], obstructive sleep apnoea (OSA) is now recognised as promoting cancer development. Although experimental $[2,3]$ and clinical $[4,5]$ evidence for this association appeared only a few years ago [6,7], rapid advances have been made in the gap of knowledge regarding the types of cancers promoted by OSA and the oncogenic pathways involved.

A recent retrospective study on 34402 patients observed that overall cancer incidence was increased after OSA diagnosis and identified a specific link with melanoma, kidney, uterine and breast cancers [8]. The association between OSA and cutaneous melanoma has been confirmed by numerous preclinical and clinical studies showing that OSA promotes its incidence $[9,10]$, progression $[2,11]$, aggressiveness [11-13] and metastatic potential $[3,14]$. In view of the poor 5-year survival rate of metastatic cutaneous melanoma [15], there is a strong need to characterise the association between OSA and cutaneous melanoma. This might allow identification of new prognostic factors for cutaneous melanoma progression and metastasis and open new avenues for innovative drugs and personalised therapies by targeting OSA as an important associated condition.

In the current issue of the European Respiratory Journal, CuBILLos-ZAPATA et al. [16] propose soluble programmed cell death ligand 1 (sPD-L1) as a potential biomarker of cutaneous melanoma aggressiveness and metastasis in OSA patients. This is quite relevant in view of the major role of the programmed cell death receptor 1 (PD-1)/PD-L1 axis in melanoma development $[15,17]$, aggressiveness [18] and survival [19], but also because of its upregulation by intermittent hypoxia and by the hypoxia-inducible factor (HIF)-1 transcription factor in cutaneous melanoma cells (figure 1) [20-22].

Indeed, among the intermediary mechanisms associated with OSA (i.e. oxidative stress, sympathetic overactivity, inflammation and intermittent hypoxia), chronic intermittent hypoxia and HIF-1 activation are now widely recognised as the main contributors to deleterious cardiometabolic consequences $[1,23]$. 
a)

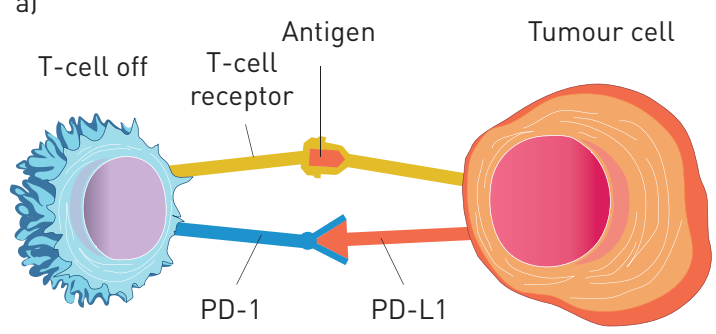

b)

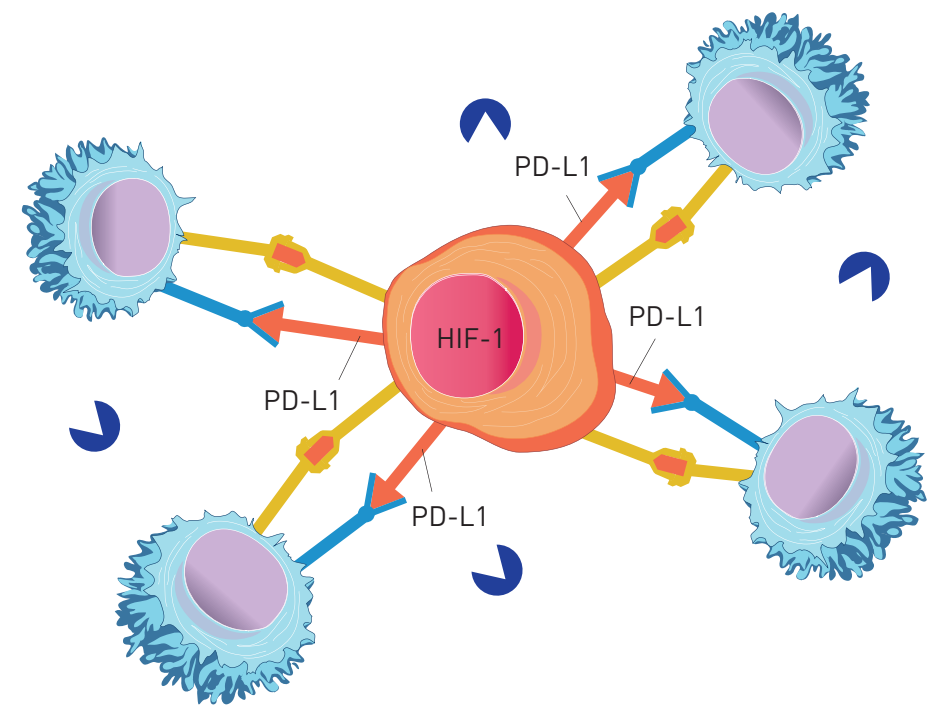

c)

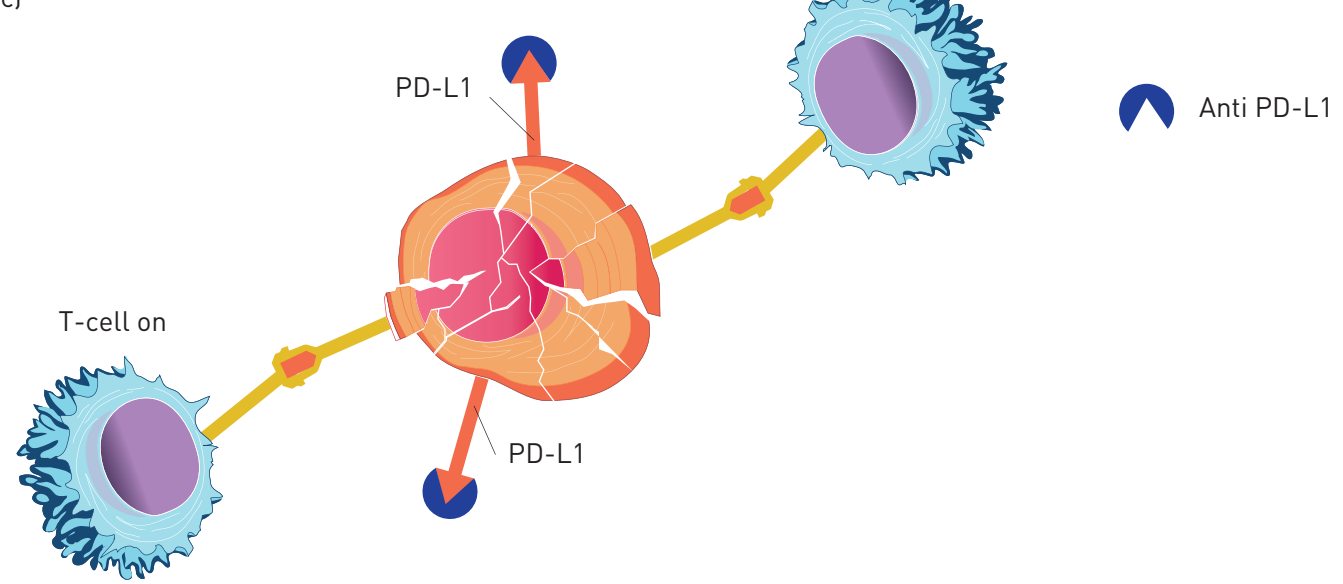

FIGURE 1 a) Immune checkpoint inhibition: binding of programmed cell death ligand 1 (PD-L1) on melanoma tumour cells to programmed cell death receptor 1 (PD-1) on T-cells inhibits their cytotoxic activity. b) Obstructive sleep apnoea (OSA)-related intermittent hypoxia promotes hypoxia-inducible factor (HIF)-1 activation and PD-L1 expression, resulting in increased tumour cell resistance to destruction mediated by T-cells and immune checkpoint inhibitors (such as anti-PD-L1). c) Proposed hypothesis behind the beneficial effects of continuous positive airway pressure treatment following OSA diagnosis: tumour cell death due to reduced PD-L1 expression with increased T-cell activity and increased anti-PD-L1 treatment efficacy.

Moreover, intermittent hypoxia and the related HIF-1 activation have also been shown to promote cancer development in vitro $[11,21,24]$ and in vivo $[2,3,10,11,14,24]$.

\section{The PD-1/PD-L1 immune checkpoint in melanoma aggressiveness}

Advanced forms of cutaneous melanoma are known to be very aggressive and it is now well established that immunomodulation plays a key role in the mechanisms behind resistance to conventional cancer therapies [15]. This evidence stems from the seminal work of James P. Allison and Tasuku Honjo, for which they were awarded the 2018 Nobel prize in Medicine and Physiology, showing that the cytotoxic T-lymphocyte-associated protein 4 (CTLA-4) and the PD-1 pathways function as molecular "brakes" on the immune system [25]. Expression of surface antigens such as PD-L1 by melanoma cells and subsequent binding to cytotoxic T-cell PD-1 in the tumour microenvironment suppresses T-cell activity, leading to inhibition of antitumoral immune responses (figure 1) [25]. Therefore, "immune checkpoint" complexes such as PD-1/PD-L1 represent ideal targets for the development of prognostic and therapeutic tools for the management of cutaneous melanoma.

Advanced cutaneous melanoma is one of the first cancers where adjuvant immunotherapies targeting the immune checkpoint have been tested [15]. Chronologically, the first adjuvant immune checkpoint blocker 
used was the CTLA-4-blocking antibody ipilimumab. Then, two PD-1 inhibitors, nivolumab and pembrolizumab, were rapidly introduced and routinely used in patients with metastatic melanoma, including those refractive to CTLA-4 blockade [25]. In a few years, adjuvant treatment strategies, including antibodies targeting the PD-1/PD-L1 axis in advanced melanoma, have thus significantly contributed to improvement of global survival and healing of cutaneous melanoma patients. In 2018, nivolumab was positioned as the new standard of care in advanced melanoma.

Regarding the value of PD-L1 as a prognostic marker of cancer evolution, recent data have shown that increased PD-L1 expression is associated with unfavourable outcome in various cancers, but there are conflicting reports regarding its prognostic value in others, such as colorectal cancer [26]. This discrepancy can be attributed to technical issues but also to the origin and form of PD-L1 investigated.

For instance, expression of PD-L1 by tumour cells and by tumour-infiltrating immune cells has opposite effects on colorectal cancer aggressiveness and metastasis [26]. Moreover, studies investigating the role of PD-L1 on cancer development should distinguish membrane PD-L1 expressed by tumour and immune cells from plasma sPD-L1 and other circulating PD-L1 sources [27]. Regarding melanoma, ZHou et al. [28] recently demonstrated that sPD-L1 could be used as a prognostic marker of metastatic potential and response to immune checkpoint treatment. Interestingly, an even more recent study has shown the same prognostic value of PD-L1-containing exosomes produced by melanoma cells [29]. This is of particular importance since exosomal PD-L1, in contrast to sPD-L1, closely reflects PD-L1 expression by tumour and metastatic cells.

\section{Why should SPD-L1 be a particularly reliable biomarker of melanoma aggressiveness and metastasis in the context of OSA?}

In their multicentre cross-sectional observational study including 360 patients with newly diagnosed cutaneous melanoma, Cubillos-ZAPATA et al. [16] compared plasma sPD-L1 levels according to the presence and severity of OSA, and evaluated relationships with cutaneous melanoma aggressiveness and invasiveness [16]. Patients were divided into four groups (non-OSA, mild OSA, moderate OSA and severe OSA) according to their apnoea-hypopnoea index (AHI) assessed by overnight respiratory polygraphy. Well-established markers were used to evaluate cutaneous melanoma aggressiveness (mitotic index, tumour ulceration and stage) and invasiveness (Breslow index, Clark levels and sentinel lymph node positivity). An overall analysis of the cohort showed that sPD-L1 levels were higher in severe OSA versus mild or non-apnoeic patients and that they were positively and independently correlated with the AHI, both in the whole cohort and in OSA patients. However, there was a huge overlap between groups and correlations were weak, questioning the specificity of the relationship.

Selective analysis of OSA patients revealed that the highest SPD-L1 levels were found in patients with poor prognostic profiles. More specifically, increased sPD-L1 levels were associated with melanoma aggressiveness markers, such as high extension stage, deep tumour invasion, tumour ulceration and sentinel lymph node involvement. None of these results were observed in non-apnoeic patients, but the authors acknowledge that this could be due to the limited sample size of the non-OSA group.

Moreover, the value of sPD-L1 as a prognostic biomarker of aggressiveness and metastasis in OSA patients was confirmed by the net improvement provided by its incorporation in classical prediction models, and in particular in the positive sentinel lymph node risk prediction model, which led to a better classification of $27.3 \%$ of the cases.

In view of these results, the study by CuBiLlos-ZAPATA et al. [16] does indeed allow proposal of sPD-L1 as a potential biomarker of cutaneous melanoma aggressiveness and metastasis in OSA patients. This is also reinforced by the fact that PD-L1 expression is upregulated by intermittent hypoxia, a major hallmark of OSA, as previously demonstrated by the same group [20] and by others [22, 30]. In fact, PD-L1 has been formally identified as a target gene of HIF-1 [22], a transcription factor known to be involved in the deleterious cardiometabolic outcome of intermittent hypoxia/OSA [23] but also to worsen cutaneous melanoma prognosis [21,30].

One of the major issues is to explore why some OSA patients develop more aggressive cutaneous melanoma than non-OSA patients. Studies have already shown that OSA is independently associated with increased aggressiveness of cutaneous melanoma $[12,13]$. Moreover, intermittent hypoxia upregulates the PD-1/PD-L1 pathway in severe OSA patients without cancer, resulting in immunomodulation of CD8 ${ }^{+}$ T-cell activity (figure 1) [20]. Such an effect of intermittent hypoxia on the PD-1/PD-L1 axis in a melanoma ecosystem may contribute to the deleterious effect of PD-L1 on cutaneous melanoma prognosis. Indeed, PD-L1 expression has been directly related to cutaneous melanoma aggressiveness [17$19,29,31]$. A major issue in the field is understanding why some patients with cutaneous melanoma are non-responders to immune checkpoint inhibitors. Approximately $60 \%$ of patients show de novo resistance 
to immune checkpoint inhibition and $20-30 \%$ of initial responders will eventually develop acquired resistance [32]. Deciphering the confounding role of intermittent hypoxia in this specific subgroup of patients might be a next step.

Finally, the study of CuBILlos-ZaPATA et al. [16] is certainly important and by far the largest prospective multicentre study in the field, and the authors should be congratulated for this. However, as acknowledged by the authors themselves, sleep fragmentation, a major confounding mechanistic factor, has not been assessed in the current study. Such an evaluation is required in future studies. Moreover, sPD-L1 elevation does not demonstrate causality; that would require interventional studies. A research agenda might be anticipated as follows: 1) an OSA prevalence study in non-responders to immune checkpoint inhibitors; 2) randomised controlled trials comparing acute impact of several weeks of sham versus effective continuous positive airway pressure (CPAP) on SPD-L1 would be highly informative; 3 ) in case of positive results, CPAP should be tested as a combined therapy for improving the long-term control of cutaneous melanoma (figure 1); and 4) finally, in view of its secretion by tumour and metastatic cells, assay of exosomal PD-L1 [29] should be tested in OSA to potentiate patient stratification.

Due to the complexity and costs of such a research programme, an international consortium is highly desirable. After crossing all these "research checkpoints", our wish is that the true impact of OSA and intermittent hypoxia on immunomodulation of cutaneous melanoma will be established and that the results will be of profit to melanoma patients.

Conflict of interest: None declared.

\section{References}

1 Lévy P, Kohler M, McNicholas WT, et al. Obstructive sleep apnoea syndrome. Nat Rev Dis Primers 2015; 1: 15015.

2 Almendros I, Montserrat JM, Ramírez J, et al. Intermittent hypoxia enhances cancer progression in a mouse model of sleep apnoea. Eur Respir J 2012; 39: 215-217.

3 Almendros I, Montserrat JM, Torres M, et al. Intermittent hypoxia increases melanoma metastasis to the lung in a mouse model of sleep apnea. Respir Physiol Neurobiol 2013; 186: 303-307.

4 Nieto FJ, Peppard PE, Young T, et al. Sleep-disordered breathing and cancer mortality: results from the Wisconsin Sleep Cohort Study. Am J Respir Crit Care Med 2012; 186: 190-194.

5 Campos-Rodriguez F, Martinez-Garcia MA, Martinez M, et al. Association between obstructive sleep apnea and cancer incidence in a large multicenter Spanish cohort. Am J Respir Crit Care Med 2013; 187: 99-105.

6 Martínez-García MÁ, Campos-Rodríguez F, Farré R. Sleep apnoea and cancer: current insights and future perspectives. Eur Respir J 2012; 40: 1315-1317.

7 Lévy P, Godin-Ribuot D, Pepin JL. Sleep apnoea and cancer: the new challenge. Eur Respir J 2014; 43: 1567-1570.

8 Sillah A, Watson NF, Schwartz SM, et al. Sleep apnea and subsequent cancer incidence. Cancer Causes Control 2018; 29: 987-994.

9 Gozal D, Ham SA, Mokhlesi B. Sleep apnea and cancer: analysis of a nationwide population sample. Sleep 2016; 39: 1493-1500.

10 Gallego-Martin T, Farré R, Almendros I, et al. Chronic intermittent hypoxia mimicking sleep apnoea increases spontaneous tumorigenesis in mice. Eur Respir J 2017; 49: 1602111.

11 Almendros I, Wang Y, Becker L, et al. Intermittent hypoxia-induced changes in tumor-associated macrophages and tumor malignancy in a mouse model of sleep apnea. Am J Respir Crit Care Med 2014; 189: 593-601.

12 Martínez-García MÁ, Martorell-Calatayud A, Nagore E, et al. Association between sleep disordered breathing and aggressiveness markers of malignant cutaneous melanoma. Eur Respir J 2014; 43: 1661-1668.

13 Martinez-Garcia MA, Campos-Rodriguez F, Nagore E, et al. Sleep-disordered breathing is independently associated with increased aggressiveness of cutaneous melanoma: a multicenter observational study in 443 patients. Chest 2018; 154: 1348-1358.

14 Li L, Ren F, Qi C, et al. Intermittent hypoxia promotes melanoma lung metastasis via oxidative stress and inflammation responses in a mouse model of obstructive sleep apnea. Respir Res 2018; 19: 28.

15 Schadendorf D, van Akkooi ACJ, Berking C, et al. Melanoma. Lancet 2018; 392: 971-984.

16 Cubillos-Zapata C, Martínez-García MÂ, Campos-Rodríguez F, et al. Soluble PD-L1 is a potential biomarker of cutaneous melanoma aggressiveness and metastasis in obstructive sleep apnoea patients. Eur Respir J 2019; 53: 1801298.

17 Merelli B, Massi D, Cattaneo L, et al. Targeting the PD1/PD-L1 axis in melanoma: biological rationale, clinical challenges and opportunities. Crit Rev Oncol Hematol 2014; 89: 140-165.

18 Audrito V, Serra S, Stingi A, et al. PD-L1 up-regulation in melanoma increases disease aggressiveness and is mediated through miR-17-5p. Oncotarget 2017; 8: 15894-15911.

19 Massi D, Brusa D, Merelli B, et al. PD-L1 marks a subset of melanomas with a shorter overall survival and distinct genetic and morphological characteristics. Ann Oncol 2014; 25: 2433-2442.

20 Cubillos-Zapata C, Avendaño-Ortiz J, Hernandez-Jimenez E, et al. Hypoxia-induced PD-L1/PD-1 crosstalk impairs T-cell function in sleep apnoea. Eur Respir J 2017; 50: 1700833.

21 Yoon DW, So D, Min S, et al. Accelerated tumor growth under intermittent hypoxia is associated with hypoxia-inducible factor-1-dependent adaptive responses to hypoxia. Oncotarget 2017; 8: 61592-61603.

22 Barsoum IB, Smallwood CA, Siemens DR, et al. A mechanism of hypoxia-mediated escape from adaptive immunity in cancer cells. Cancer Res 2014; 74: 665-674.

23 Belaidi E, Morand J, Gras E, et al. Targeting the ROS-HIF-1-endothelin axis as a therapeutic approach for the treatment of obstructive sleep apnea-related cardiovascular complications. Pharmacol Ther 2016; 168: 1-11. 
24 Campillo N, Torres M, Vilaseca A, et al. Role of cyclooxygenase-2 on intermittent hypoxia-induced lung tumor malignancy in a mouse model of sleep apnea. Sci Rep 2017; 7: 44693.

25 Wolchok J. Putting the immunologic brakes on cancer. Cell 2018; 175: 1452-1454.

26 Lee KS, Kim BH, Oh HK, et al. Programmed cell death ligand-1 protein expression and CD274/PD-L1 gene amplification in colorectal cancer: implications for prognosis. Cancer Sci 2018; 109: 2957-2969.

27 Zhu X, Lang J. Soluble PD-1 and PD-L1: predictive and prognostic significance in cancer. Oncotarget 2017; 8: 97671-97682.

28 Zhou J, Mahoney KM, Giobbie-Hurder A, et al. Soluble PD-L1 as a biomarker in malignant melanoma treated with checkpoint blockade. Cancer Immunol Res 2017; 5: 480-492.

29 Chen G, Huang AC, Zhang W, et al. Exosomal PD-L1 contributes to immunosuppression and is associated with anti-PD-1 response. Nature 2018; 560: 382-386.

30 Almendros I, Martínez-García MÁ, Campos-Rodríguez F, et al. Intermittent hypoxia is associated with high hypoxia inducible factor- $1 \alpha$ but not high vascular endothelial growth factor cell expression in tumors of cutaneous melanoma patients. Front Neurol 2018; 9: 272.

31 Chen L, Han X. Anti-PD-1/PD-L1 therapy of human cancer: past, present, and future. J Clin Invest 2015; 125: 3384-3391.

32 Syn NL, Teng MWL, Mok TSK, et al. De-novo and acquired resistance to immune checkpoint targeting. Lancet Oncol 2017; 18: e731-e741. 